\title{
Optimal Frequency of Operation and Radiation Efficiency Limitations of Implantable Antennas
}

\author{
Denys Nikolayev* $^{* \dagger}$, Zvonimir Sipus ${ }^{\ddagger}$, Marko Bosiljevac ${ }^{\ddagger}$, Wout Joseph ${ }^{\S}$, \\ Maxim Zhadobov ${ }^{\dagger}$, Ronan Sauleau ${ }^{\dagger}$, Luc Martens ${ }^{\S}$, Anja K. Skrivervik* \\ *Microwave and Antenna Group (MAG), École polytechnique fédérale de Lausanne, \\ CH-1015 Lausanne, Switzerland \\ †Univ Rennes, CNRS, Institut d’Électronique et de Télécommunications de Rennes, UMR-6164, \\ FR-35000 Rennes, France \\ ${ }^{\ddagger}$ Faculty of Electrical Engineering and Computing, University of Zagreb, HR-10000 Zagreb, Croatia \\ $\S$ INTEC - WAVES, imec / Ghent University, BE-9052 Ghent, Belgium \\ d@deniq.com
}

\begin{abstract}
Fundamental limits on radiation performance of implantable antennas serve as the design quality gauge, facilitate the choice of the antenna type, and provide simple design rules to maximize the radiation performance. This study obtains the limits using two formulations: 1) theoretical spherical-wave expansion using elementary magnetic and electric dipoles and 2) realistic full-wave $\mathbf{2 D}$-axisymmetric models of $\mathbf{T} \mathbf{M}_{10}$ and $\mathbf{T E} \mathbf{E}_{10}$ mode capsule antennas. Using both formulations, the optimal radiation conditions are investigated, the effects of antenna dimensions and its implantation depth are quantified. The results also demonstrate that an electric antenna operating close to the optimal frequency could achieve higher efficiency than a magnetic one. The latter, however, is more efficient below the optimal frequency range.
\end{abstract}

Index Terms-fundamental limits, radiation efficiency, implantable antennas, spherical wave expansion.

\section{INTRODUCTION}

Wireless, miniature, and autonomous implantable devices enable many potential breakthroughs in medicine, clinical research, and basic science [1]-[3]. Such devices require heavily miniaturized and integrated antennas to communicate with external on- or off-body systems. A variety of antennas has been proposed for implantable and ingestible capsule applications [5]-[18]. However, establishing efficient links with implants remains a major challenge because of too low radiation efficiencies $(\eta<0.1 \%)$ of the antennas operating in lossy, dispersive, and heterogeneous tissues [3]. Moreover, the exact location of the implant is often uncertain. Considering typical antenna input power from a few to about a hundred of $\mathrm{mW}$ (limited by safety regulations and standards) and Rx sensitivities, this efficiency provides an operating range up to only a few meters [3]. Fundamental studies are therefore required to investigate on how to improve the radiation efficiency of implantable antennas.

Establishing the fundamental limitations on radiation performance could serve in practice as the design quality gauge, facilitate the choice of the antenna type and dimensions, and

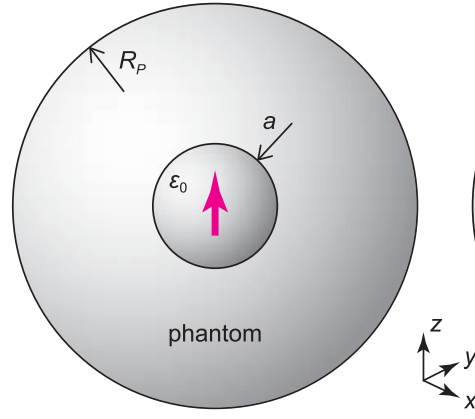

(a)

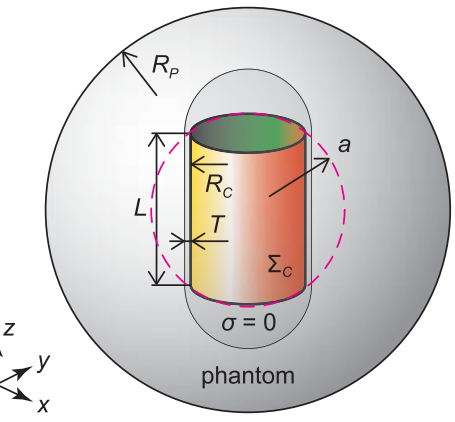

(b)
Fig. 1. Problem formulation: the sources are centered inside of a dispersive muscle-equivalent phantom $\varepsilon_{r}(f), \sigma(f)$ of radius $R_{P}$ (not to scale). (a) Theoretical spherical-wave expansion model: elementary electric (TM) or magnetic (TE) dipoles surrounded by an air bubble of radius $a$. (b) Realistic model of a capsule-conformal antenna: current density distributions on a finitesized cylindrical surface $\Sigma_{C}$ of circumradius $a$ represent equivalent source.

provide simple design rules to maximize the efficiency. Impact of tissues on radiation performance has been considered in [19], [20] for small inductor sources and in [21] for infinitesimal magnetic and electric sources. Finite-size $\mathrm{TM}_{10}$ and $\mathrm{TE}_{10}$ sources were considered in [22]. In this study, we determine the optimal radiating conditions of magnetic and electrical sources and their achievable efficiencies using a theoretical (spherical-wave expansion) and a realistic (fullwave) formulations of the problem.

\section{Problem Formulation}

The sources in both formulations are centered inside of a spherical phantom of radius $R_{P}$ (Fig. 1) with dispersive muscle-equivalent EM properties according to [23]. Spherical model of human body provides worthwhile and useful results despite being only a rough approximation [21], [22]. Such shape introduces isotropic, direction-independent losses con- 
serving the intrinsic radiation pattern independently of the antenna orientation [10]. Therefore, it could serve as a reference, well-characterized phantom improving the reproducibility of the results and allowing to gauge antenna designs relatively to the maximum achievable efficiency for the established reference case.

\section{A. Theoretical Spherical Wave Expansion Model}

The analyzed structure, shown in Fig. 1a, contains a spherical phantom and an implantable antenna. The antenna is modeled as a small sphere of radius $a$ (filled with air) with a current source centered inside (either an electric or a magnetic dipole).

The solution uses the spherical-wave modal expansion (SWE) methodology. The EM field in a spherical structure (with zero free-charge density) can be accurately represented using vector spherical harmonics [24] as

$$
\mathbf{E}=-\sum_{n} \sum_{m} a_{m n} \mathbf{M}_{m n}+b_{m n} \mathbf{N}_{m n}
$$

where

$$
\begin{aligned}
\mathbf{M}_{m n} & =\nabla \times \hat{\mathbf{r}} \psi_{m n}, \mathbf{N}=\frac{1}{k} \nabla \times \mathbf{M}_{m n}, \text { and } \\
\psi_{m n} & =\mathbf{z}_{n}(k r) P_{n}^{m}(\cos \theta) e^{j m \varphi} .
\end{aligned}
$$

Here, $\psi_{m n}$ is the solution to the Helmholtz equation. Specifically, $\mathbf{z}_{n}$ stands for spherical Bessel or Hankel functions.

The EM fields in the source sphere and in the outer sphere (human body) are matched using Love's equivalence theorem [25]. Therefore, for the equivalent inner problem, we consider the radiation of a dipole and of equivalent currents in the free-space. For the equivalent outer problem, we consider equivalent currents radiating in the homogeneous space with the permittivity equal to the permittivity of the layer that surrounds the inner "source" sphere. The spherical-harmonic representations of these two equivalent problems fulfill the boundary condition of the continuous tangential EM-field at the boundary of the inner sphere. The phantom-free-space boundary is included in the outside equivalent problem using the scattered field approach.

Finally, using the orthogonality property of spherical harmonics [24], we obtain a linear system of equations containing $2 N_{\text {mode }}$ unknowns, where $N_{\text {mode }}$ is the number of spherical modes in the modal representation of EM field. Note that the considered analysis approach can also be applied to implantable antennas with off-center position [25].

\section{B. Realistic Full-Wave 2D-Axisymmetric Model}

In realistic model, the equivalent-source surface $\Sigma_{C}$ is defined by a cylinder of the variable length $L$ and radius $R_{C}$ (Fig. 1b). In this study, we constrain $R_{C}=3 L / 8$ to preserve the source proportions while varying the circumradius $a \equiv \sqrt{L^{2} / 4+R_{C}^{2}}$. This is done to improve the coherence with the theoretical model (see Section II-A). To represent a generic capsule device, a lossless $(\sigma=0)$ region encloses the surface $\Sigma_{C}$. The variable $T$ allows for evaluating the impact of shell

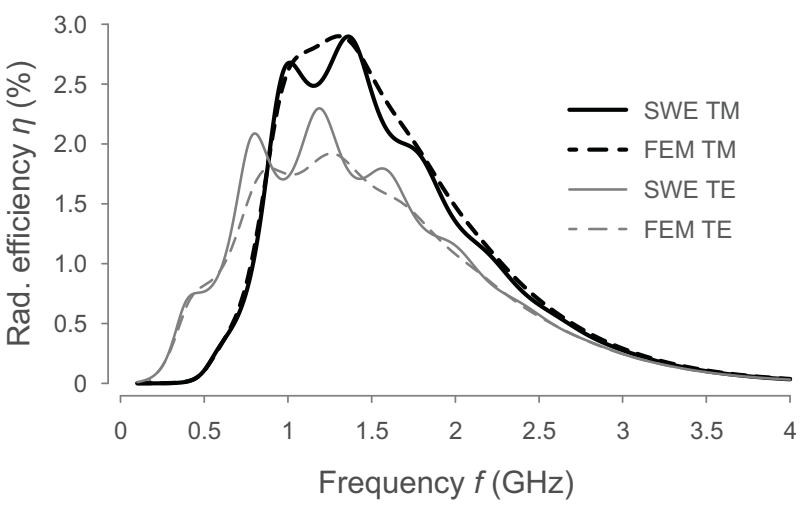

Fig. 2. Baseline results: the maximum achievable radiation efficiency $\eta(f)$ of electric (TM) and magnetic (TE) sources of $a=10 \mathrm{~mm}$ in the canonical $R_{P}=5 \mathrm{~cm}$ muscle-equivalent phantom. The results are computed for two model formulations: 1) theoretical spherical-wave expansion (SWE; Fig. 1a) and 2) realistic full-wave 2D axisymmetric (FEM; Fig. 1b).

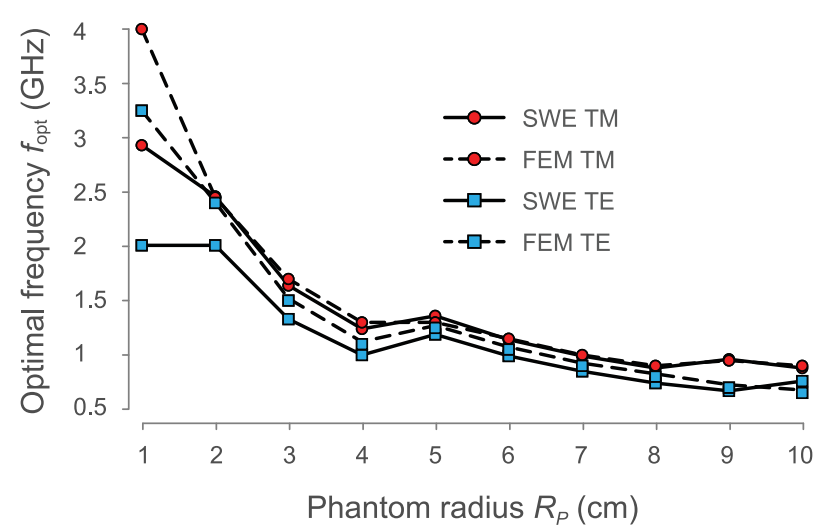

(a)

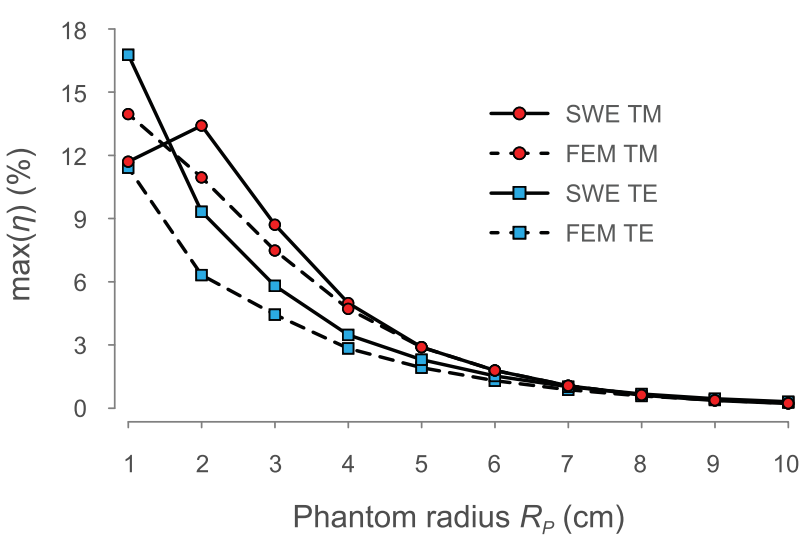

(b)

Fig. 3. Effect of the phantom size $R_{P}$ (asymptotic to the antenna implantation depth) on (a) the optimal frequency of operation $f_{\text {opt }}$ [defined as $\eta\left(f_{\text {opt }}\right) \equiv$ $\max (\eta)$ ] and (b) the maximum achievable radiation efficiency $\max (\eta)$. Both $f_{\text {opt }}$ and $\max (\eta)$ decay exponentially with the implantation depth. 
(or superstrate) thickness on $\eta$. It is assumed that the model contains only non-magnetic materials.

The surface current densities $\mathbf{J}_{s}(r, \varphi, z)$ are defined on $\Sigma_{C}$ for $\mathrm{TM}_{10}$ and $\mathrm{TE}_{10}$ modes as

$$
\begin{aligned}
\mathbf{J}_{s, \mathrm{TM}_{10}} & =[0,0, \cos (\pi z / L)], \\
\mathbf{J}_{s, \mathrm{TE}_{10}} & =(0,1,0) .
\end{aligned}
$$

EM field radiated from a realistic equivalent source inside of dispersive phantom satisfies the inhomogeneous wave equation. In terms of the time-harmonic $\mathbf{E}$ (i.e. time variation $\left.e^{i \omega t}\right)$, it could be expressed as $\nabla^{2} \mathbf{E}=j \omega \mu_{0} \mathbf{J}_{s}+$ $j \omega \mu_{0} \sigma \mathbf{E}-\omega^{2} \mu_{0} \varepsilon_{0} \varepsilon_{r} \mathbf{E}$, where $\mathbf{J}_{s}$ is the imposed electric current density [24]. Taking into account the $z$-axial symmetry of the problem on Fig. $1 \mathrm{~b}$, we reduce it to $\mathbb{R}^{2}$ assuming $\mathbf{E}(r, \varphi, z)=\overline{\mathbf{E}}(r, z) e^{-i m \varphi}$, where $m$ is the azimuthal mode number.

The radiation efficiency is obtained using the Poynting's theorem [24] as $\eta \equiv \Re\left(P_{e}\right) / \Re\left(P_{s}\right)$, where the exiting power $P_{e}$ and the supplied power $P_{s}$ are evaluated as in [22]. Note that for a realistic in-body antenna, the total radiation efficiency also includes a mismatch loss as $\eta_{t o t}=\eta\left(1-\left|\Gamma_{A}\right|^{2}\right)$, where $\Gamma_{A}$ is the reflection coefficient at the antenna feed. Here, we consider $\Gamma_{A}=0$. The mismatch-loss immunity of in-body antennas was addressed previously in [16].

Finally, the solution of the governing wave equation can be rapidly obtained numerically with, for instance, a fullyadaptive $h p$-FEM (finite element method) implemented in Agros2D [26]. Alternatively, the commercial codes such as COMSOL [27] could be used.

\section{RESUlTS AND DISCUSSION}

Both formulations of the problem reveal that the radiation efficiency $\eta$ of TM and TE sources has a skew-normal distribution shape as Fig. 2 shows. The peak $\max (\eta)$ defines the optimal operating frequency $f_{\text {opt }}$ that-for a reference case of $R_{P}=5, a=1 \mathrm{~cm}$-occupies the $f \approx[1,1.5] \mathrm{GHz}$ range. This is consistent with previous findings for different source formulations [19]-[22]. At the optimal frequency of operation, $\eta$ peaks at about $3 \%$. Compared to existing antenna designs with the reported efficiencies around $\eta \in\left[10^{-5}, 10^{-4}\right]$, the results show that there is a potential of about an order of magnitude of radiation performance improvement. This can be achieved in part by a proper choice of the antenna type and operating frequency. As Fig. 2 shows, magnetic antennas could be more appropriate for lower bands $(f<0.9 \mathrm{GHz})$ that includes various MedRadio/MICS 401-457 MHz bands and the Industrial, Scientific and Medical (ISM) $434 \mathrm{MHz}$ band. Starting from the ISM $915 \mathrm{MHz}$ band, electric antennas could provide higher efficiencies despite the increased nearfield losses in surrounding tissues [21]. High permittivity of the surrounding tissues $\left(\varepsilon_{r} \gtrsim 50\right)$ loads the electric antennas therefore significantly increasing their electrical size $k a \propto \max (\eta)$ [28]. This effect could be further exploited by using highpermittivity insulating layers (superstrates) [29]. This approach has been recently demonstrated in practice in [13] using a lowloss ceramic capsule shell with $\varepsilon_{r} \approx 80$.

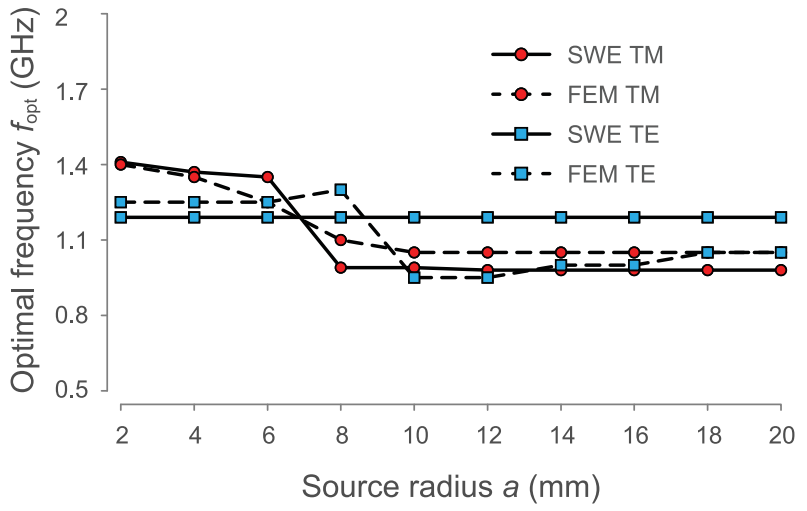

(a)

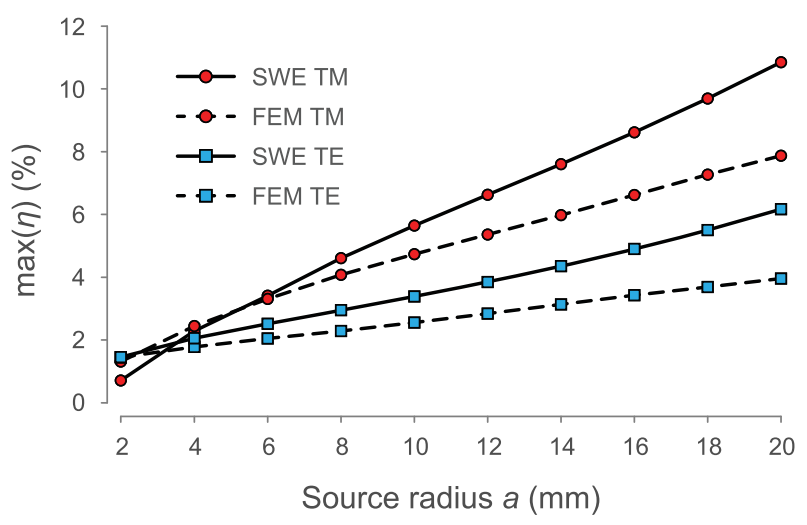

(b)

Fig. 4. Effect of the source size $a$ on (a) the optimal frequency of operation $f_{\text {opt }}$ and (b) the maximum achievable radiation efficiency $\max \eta\left(R_{P}=\right.$ $5 \mathrm{~cm}$ ). Whereas $f_{\text {opt }}$ remains reasonably invariant to the source size, $\max (\eta)$ is strongly dependent on it. Both model formulations show stronger effect for TM sources.

The results shown in Fig. 2 are strongly dependent on the phantom size (asymptotic to the implantation depth of the antenna) and the source circumradius $a$. We study the effect of the phantom by varying $R_{P} \in[1,10] \mathrm{cm}$. Clearly, bigger phantom implies deeper antenna implantation, which results in increased power dissipation and thus lower efficiency. Increased power dissipation means also that a lower frequency of operation would be preferable since the penetration depth is increased. Fig. 3 quantifies these effects. Both the optimal frequency and the maximum efficiency decay exponentially with the implantation depth. The agreement between the theoretical and realistic models is exceptional for larger phantoms $\left(R_{P}>\right.$ $4 \mathrm{~cm})$. For the smaller phantoms, the difference in source formulation becomes noticeable. However, even at $R_{P}=1 \mathrm{~cm}$ (subcutaneous implantation), the predicted values are adequate and useful: $f_{\text {opt }} \in[2,4] \mathrm{GHz}, \max (\eta) \in[12,17] \%$.

The physical size $a$ of the source has an mild effect on $f_{\text {opt }}$. As Fig. 4a shows, the optimal frequency shifts from 1.21.4-GHz range to $\approx 0.9 \mathrm{GHz}$. SWE approximation shows no effect of $a$ on $f_{\text {opt }}$ of TE source. This could be related to the number of harmonics under consideration but requires further 
investigation.

The maximum achievable $\eta$ depends strongly on the source size $a$. Results in Fig. 4b demonstrate a linear dependence of $\max [\eta(a)]$ and the effect is stronger for TM sources. Note that for all $a$ except $a<3 \mathrm{~mm}$, electric TM sources provide higher $\max (\eta)$ for implantable applications according to both problem formulations. For the realistic formulation, the strongest effect comes from the source length $L$ (see Fig. 1b). Here, the maximum achievable efficiency could be accurately represented for both operating modes $\left(\bar{R}^{2}=0.9725\right.$ for TM mode and $\bar{R}^{2}=0.9769$ for TE mode, where $\bar{R}^{2}$ is the adjusted coefficient of determination):

$$
\max (\eta)=\frac{3 c_{1} \ln \left(f+c_{2}\right)}{L} \exp \left(-\frac{\ln L-1 / 3}{2 c_{4}^{3}}\right),
$$

where $L$ is the length in $\mathrm{mm}, f$ is the frequency in $\mathrm{GHz}$, and $c_{i}$ are the mode-dependent coefficients. For TM mode: $c_{1}=0.00318, c_{2}=59.82, c_{3}=0.532$, and for TE: $c_{1}=$ $0.00675, c_{2}=1.252, c_{3}=0.4$.

\section{CONCLUSION}

When an implantable antenna operates at the optimal frequency of operation, the dissipated energy is minimized for a given power flow in the far field. The minimum of dissipated energy defines is equivalent to the maximum achievable radiation efficiency. In this study, we determined the optimal radiating conditions of magnetic and electrical sources and their achievable efficiencies using a theoretical (spherical-wave expansion) and a realistic (full-wave) models. The established efficiency limitations serve in practice as the design quality gauge, facilitate the choice of the source type and its dimensions according to a given application, and provide simple rules to verify the feasibility of a specified design. The results show that the derived limitations exceed the efficiencies of conventional designs by about an order on magnitude. This can be achieved in part by a proper choice of the antenna type and operating frequency. Future work on this subject involves investigating and optimizing other antenna shapes, considering and quantifying in statistical-EM terms the effects of realistic human body models.

\section{REFERENCES}

[1] E. Katz, Implantable Bioelectronics. Weinheim, Germany: Wiley, 2014.

[2] D. Nikolayev, M. Zhadobov, R. Sauleau, and P. Karban, "Antennas for ingestible capsule telemetry," in Advances in Body-Centric Wireless Communication: Applications and State-of-the-Art, London, UK: IET, 2016, pp. 143-186.

[3] A. Kiourti and K. S. Nikita, "A review of in-body biotelemetry devices: implantables, ingestibles, and injectables," IEEE Trans. Biomed. Eng., vol. 64, no. 7, pp. 1422-1430, Jul. 2017.

[4] D. Nikolayev, M. Zhadobov, P. Karban, and R. Sauleau, "Conformal antennas for miniature in-body devices: The quest to improve radiation performance," URSI Rad. Sci. Bull., vol. 363, pp. 52-64, Dec. 2017.

[5] S. Bakogianni and S. Koulouridis, "On the design of miniature MedRadio implantable antennas," IEEE Trans. Antennas Propag., vol. 65, no. 7, pp. 3447-3455, Jul. 2017.

[6] Z. Jiang et al., "Wideband loop antenna with split ring resonators for wireless medical telemetry," IEEE Antenn. Wireless Propag. Lett., vol. 18, no. 7, pp. 1415-1419, Jul. 2019.
[7] W. Lei and Y. Guo, "Design of a dual-polarized wideband conformal loop antenna for capsule endoscopy systems," IEEE Trans. Antennas Propag., vol. 66, no. 11, pp. 5706-5715, Nov. 2018.

[8] F. Merli et al., "Design, realization and measurements of a miniature antenna for implantable wireless communication systems," IEEE Trans. Antennas Propag., vol. 59, no. 10, pp. 3544-3555, Oct. 2011.

[9] M. S. Miah et al., "Antenna system design for improved wireless capsule endoscope links at $433 \mathrm{MHz}, "$ IEEE Trans. Antennas Propag., vol. 67, no. 4, pp. 2687-2699, Apr. 2019.

[10] D. Nikolayev, M. Zhadobov, L. Le Coq, P. Karban, and R. Sauleau, "Robust ultra-miniature capsule antenna for ingestible and implantable applications," IEEE Trans. Antennas Propag., vol. 65, no. 11, pp. 61076119, Nov. 2017.

[11] Y. Peng , K. Saito, and K. Ito, "Antenna design for impulse-radiobased wireless capsule endoscope communication systems," IEEE Trans. Antennas Propag., vol. 66, no. 10, pp. 5031-5042, Oct. 2018.

[12] J. Wang et al., "An implantable and conformal antenna for wireless capsule endoscopy," IEEE Antenn. Wireless Propag. Lett., vol. 17, no. 7, pp. 1153-1157, Jul. 2018.

[13] D. Nikolayev, W. Joseph, A. Skrivervik, M. Zhadobov, L. Martens, and R. Sauleau, "Dielectric-loaded conformal microstrip antennas for versatile in-body applications," IEEE Antenn. Wireless Propag. Lett., to be published. doi: 10.1109/LAWP.2019.2948814.

[14] Z. Duan, L. J. Xu, S. Gao, and W. Geyi, "Integrated design of wideband omnidirectional antenna and electronic components for wireless capsule endoscopy systems," IEEE Access, vol. 6, pp. 29626-29636, May 2018.

[15] Y. Feng, Y. Li, L. Li, B. Ma, H. Hao, and L. Li, "Tissue-dependent co-matching method for dual-mode antenna in implantable neurostimulators," IEEE Trans. Antennas Propag., vol. 67, no. 8, pp. 5253-5264, Aug. 2019

[16] D. Nikolayev, M. Zhadobov, and R. Sauleau, "Immune-to-detuning wireless in-body platform for versatile biotelemetry applications," IEEE Trans. Biomed. Circuits Syst., vol. 13, no. 2, pp. 403-412, Jan. 2019.

[17] M. K. Magill, G. A. Conway, and W. G. Scanlon, "Tissue-independent implantable antenna for in-body communications at $2.36-2.5 \mathrm{GHz}$," IEEE Trans. Antennas Propag., vol. 65, no. 9, pp. 4406-4417, Sep. 2017.

[18] Z. Bao, Y.-X. Guo, and R. Mittra, "Conformal capsule antenna with reconfigurable radiation pattern for robust communications," IEEE Trans. Antennas Propag., vol. 66, no. 7, pp. 3354-3365, Apr. 2018.

[19] S. Kim, J. S. Ho, and A. S. Y. Poon, "Midfield wireless powering of subwavelength autonomous devices," Phys. Rev. Lett., vol. 110, no. 20, p. 203905, May 2013.

[20] D. Nikolayev, M. Zhadobov, P. Karban, and R. Sauleau, "Electromagnetic radiation efficiency of body-implanted devices," Phys. Rev. Applied, vol. 9, no. 2, p. 024033, Feb. 2018.

[21] A. K. Skrivervik, M. Bosiljevac, and Z. Sipus, "Fundamental limits for implanted antennas: Maximum power density reaching free space," IEEE Trans. Antennas Propag., vol. 67, no. 8, pp. 4978-4988, Aug. 2019.

[22] D. Nikolayev, W. Joseph, M. Zhadobov, R. Sauleau, and L. Martens, "Optimal radiation of body-implanted capsules," Phys. Rev. Lett., vol. 122, no. 10, p. 108101, Mar. 2019.

[23] S. Gabriel, R. W. Lau, and C. Gabriel, "The dielectric properties of biological tissues: II. Measurements in the frequency range $10 \mathrm{~Hz}$ to 20 GHz," Phys. Med. Biol., vol. 41, pp. 2251-2269, Nov. 1996.

[24] J. D. Jackson, Classical Electrodynamics, 3rd ed. Hoboken, NJ: John Wiley \& Sons, 1999.

[25] M. Bosiljevac, Z. Sipus, and A. K. Skrivervik, "Propagation in finite lossy media: an application to WBAN," IEEE Antenn. Wireless Propag. Lett., vol. 14, pp. 1546-1549, Mar. 2015.

[26] D. Nikolayev, Z. Kubík, P. Karban, and J. Skála, "Impedance analysis of transmission line cells for EMC applications using Agros2D," Appl. Math. Comput., vol. 289, pp. 381-387, Oct. 2016.

[27] COMSOL Inc. COMSOL Multiphysics. Accessed: Oct. 16, 2019. [Online]. Available: https://www.comsol.com/

[28] D. Nikolayev, M. Zhadobov, and R. Sauleau, "Impact of tissue electromagnetic properties on radiation performance of in-body antennas," IEEE Antenn. Wireless Propag. Lett., vol. 17, no. 8, pp. 1440-1444, Aug. 2018.

[29] F. Merli, B. Fuchs, J. R. Mosig, and A. K. Skrivervik, "The effect of insulating layers on the performance of implanted antennas," IEEE Trans. Antennas Propag., vol. 59, no. 1, pp. 21-31, Jan. 2011. 